\title{
Synthesis of highly functionalized $\beta$-aminocyclopentanecarboxylate stereoisomers by reductive ring opening reaction of isoxazolines
}

\author{
Melinda Nonn ${ }^{1}$, Loránd Kiss ${ }^{1}$, Reijo Sillanpää ${ }^{2}$ and Ferenc Fülöp ${ }^{* 1,3}$
}

\section{Full Research Paper}

\section{Address:}

${ }^{1}$ Institute of Pharmaceutical Chemistry, University of Szeged, Eötvös u. 6, H-6720 Szeged, Hungary, ${ }^{2}$ Department of Chemistry, University of Jyväskylä, FIN-40014 Jyväskylä, Finland and ${ }^{3}$ Stereochemistry

Research Group of the Hungarian Academy of Sciences, University of Szeged, Eötvös u. 6, H-6720 Szeged, Hungary

\section{Email:}

Ferenc Fülöp* - fulop@pharm.u-szeged.hu

* Corresponding author

Keywords:

amino acids; cycloaddition; functionalization; isoxazolines; reduction

\author{
Beilstein J. Org. Chem. 2012, 8, 100-106. \\ doi:10.3762/bjoc.8.10 \\ Received: 05 October 2011 \\ Accepted: 23 December 2011 \\ Published: 17 January 2012 \\ Associate Editor: D. Dixon \\ () 2012 Nonn et al; licensee Beilstein-Institut. \\ License and terms: see end of document.
}

\begin{abstract}
A rapid and simple procedure was devised for the synthesis of multifunctionalized cyclic $\beta$-amino esters and $\gamma$-amino alcohols via the 1,3-dipolar cycloaddition of nitrile oxides to $\beta$-aminocyclopentenecarboxylates. The opening of the isoxazoline reductive ring to the corresponding highly functionalized 2-aminocyclopentanecarboxylates occurred stereoselectively with good yields.
\end{abstract}

\section{Introduction}

Isoxazoline-fused amino acids are important bioactive derivatives in organic and medicinal chemistry (e.g., conformationally restricted aspartate and glutamate analogues) [1-6]. As a consequence of their ability to undergo reductive ring opening, isoxazolines are of interest as precursors for the synthesis of highly functionalized molecules such as $\beta$-hydroxyketones [7-10], amino alcohols or amino acids [11-17], etc. The multifunctionalized cyclic amino acids - e.g., the antibiotic Oryzoxymycin [18-21], the antiviral agents Tamiflu [22-33], Zanamivir and 2,3-didehydro-2-deoxy- $N$-acetylneuraminic acid (DANA) [34-38] - are bioactive derivatives of great significance for medicinal chemistry. A promising neuraminidase inhibitor, BCX-1812 (Peramivir), is currently under evaluation in clinical trials [39-45] (Figure 1). A series of Peramivir analogues has recently been investigated as potential antiviral agents $[46,47]$.

\section{Results and Discussion}

We recently reported a regio- and stereoselective procedure for the formation of a series of isoxazoline-fused cispentacin and transpentacin regio- and stereoisomers (2-6) from bicyclic $\beta$-lactam 1 [48,49] (Scheme 1). The syntheses consisted of a dipolar cycloaddition of nitrile oxide (generated with $\mathrm{Boc}_{2} \mathrm{O}$, $\mathrm{Et}_{3} \mathrm{~N}$ and DMAP) to the olefinic bond of cis-ethyl 2-aminocyclopent-3-enecarboxylate derived from 1 , during which the isoxazoline-fused amino ester regio- and stereoisomers ( 2 and 

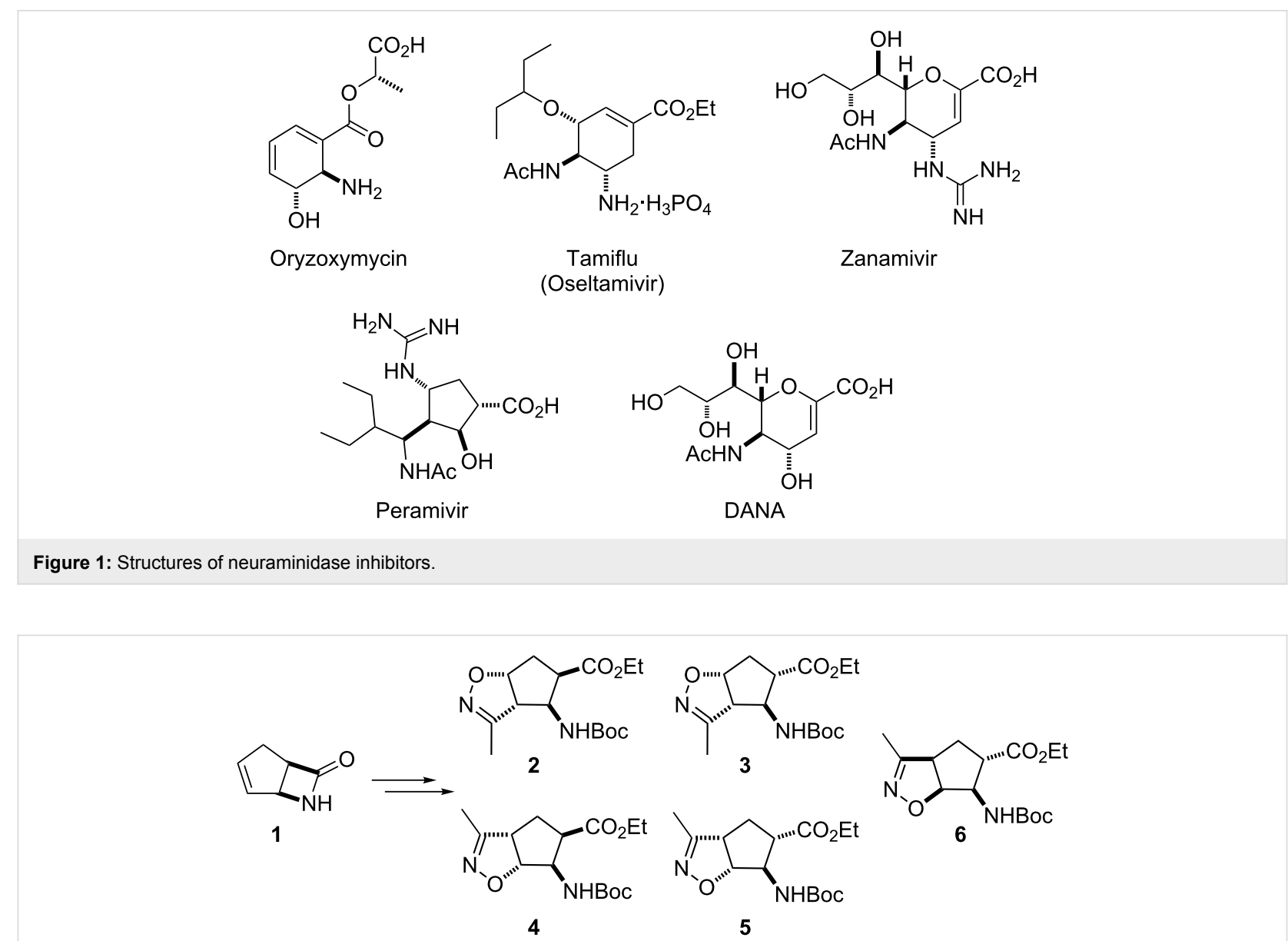

Scheme 1: Isoxazoline-fused $\beta$-aminocyclopentanecarboxylate regio- and stereoisomers [8].

4) were formed, then separated and isolated. The cycloaddition of nitrile oxide to trans-ethyl 2-aminocyclopent-3-enecarboxylate under similar conditions proceeded selectively with the formation of $\mathbf{6}$. Epimerization of $\mathbf{2}$ and $\mathbf{4}$ afforded trans derivatives $\mathbf{3}$ and $\mathbf{5}[48,49]$.

Since isoxazoline-functionalized molecules are excellent precursors for the construction of different functional groups through reductive ring cleavage, our recent aim was to synthesize highly functionalized $\beta$-aminocyclopentanecarboxylate regio- and stereoisomers from the earlier prepared isoxazolinefused cispentacin and transpentacin derivatives.

A number of methods are known for the reductive opening of the isoxazoline ring: Catalytic hydrogenation or reduction with $\mathrm{Fe}$ in the presence of $\mathrm{NH}_{4} \mathrm{Cl}, \mathrm{NaBH}_{4}, \mathrm{LiAlH}_{4}$, Raney $\mathrm{Ni}$, $\mathrm{BH}_{3} \cdot \mathrm{THF}$, or $\mathrm{SmI}_{2} / \mathrm{B}(\mathrm{OH})_{3} / \mathrm{H}_{2} \mathrm{O}$ [7-17].

For the reduction, we selected model compound $\mathbf{2}$ from earlier prepared isoxazoline-fused cispentacin stereoisomers to execute the reduction under different conditions. The isoxazoline-fused derivative was treated with the above-mentioned reducing agents. Unfortunately, neither transformation nor isoxazoline opening with ester reduction was observed. When the reduction was carried out with $\mathrm{NaBH}_{4}$ in EtOH, three products were obtained: The epimerized isoxazoline-fused amino carboxylate 7 and amino alcohols 8 and 9 which were separated by chromatography and isolated (Scheme 2).

Thus, this reaction did not lead to the formation of highly functionalized isoxazoline ring-opened $\beta$-amino ester either. When ammonium formate in $\mathrm{EtOH}$ in the presence of $\mathrm{Pd} / \mathrm{C}$ was investigated for the reduction of $\mathbf{2}$, the ring opening resulted in carbonyl compound $\mathbf{1 0}$ in rather low yield through the corresponding hydroxyimine intermediate, followed by elimination and saturation (Scheme 3).

Combinations of $\mathrm{NaBH}_{4}$ (as a mild and selective reducing agent) with cobalt, nickel, iridium or rhodium halide have previously been employed for cleavage of the isoxazoline ring system, which is otherwise inert to $\mathrm{NaBH}_{4}$ without such metal halide additives [50]. Accordingly, we investigated the reduc- 


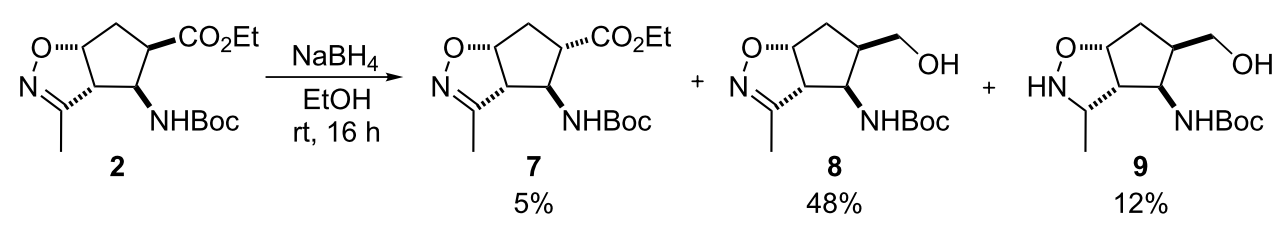

Scheme 2: Treatment of isoxazoline-fused amino ester 2 with $\mathrm{NaBH}_{4}$.

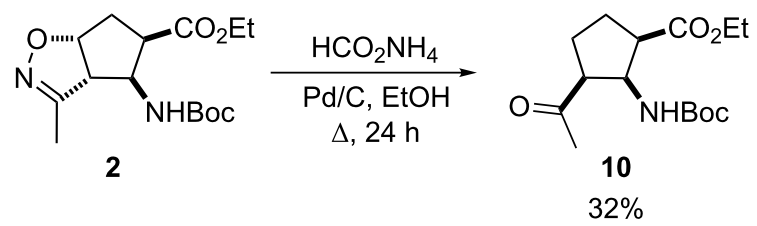

Scheme 3: Reduction with $\mathrm{Pd} / \mathrm{C}$ in the presence of $\mathrm{HCO}_{2} \mathrm{NH}_{4}$.

tion of isoxazoline-fused amino ester stereoisomers 2 [48,49] with $\mathrm{NaBH}_{4}$ in the presence of $\mathrm{NiCl}_{2}$ (Scheme 4), which was found to be a suitable reducing system.

The reduction carried out by adding $\mathrm{NaBH}_{4}$ to a mixture of $\mathrm{NiCl}_{2}$ and isoxazoline derivative 2 in $\mathrm{EtOH} / \mathrm{H}_{2} \mathrm{O}$, followed by amino group protection with $\mathrm{Boc}_{2} \mathrm{O}$, selectively afforded only isoxazoline-opened product $\mathbf{1 2}$ as a single diastereomer in good yield. The reaction was exothermic and deposited a black granular precipitate, reflecting the presence of metal boride. The product was purified by column chromatography and the structure of $\mathbf{1 2}$ was certified by X-ray analysis (Figure 2).

The isoxazoline opening occurred with the formation of a new stereocenter at a one-carbon distance from C-3. In accordance with earlier results [39-47], the hydrogenation of the isoxazoline proceeded through hydrogen attack from the carbamate side (cis to -NHBoc) of the cyclopentane skeleton. This was confirmed by X-ray analysis of $\mathbf{1 2}$.

In order to increase the number of multifunctionalized amino ester stereoisomers, we next examined the reductions of isoxazoline-fused cispentacin and transpentacin stereoisomers (3-6)

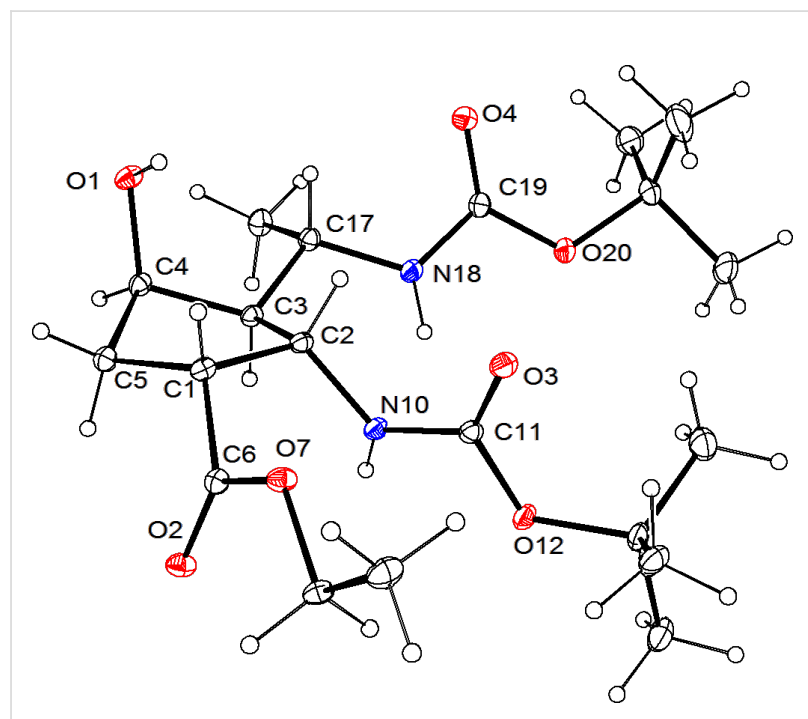

Figure 2: ORTEP diagram of 12 showing the atomic labeling scheme. The thermal ellipsoids are drawn at the $20 \%$ probability level.

[49]. Reactions were carried out similarly with $\mathrm{NaBH}_{4}$ in the presence of $\mathrm{NiCl}_{2}$ in $\mathrm{EtOH} / \mathrm{H}_{2} \mathrm{O}$ and led selectively to the corresponding multifunctionalized amino esters 13-16 in good yields (Scheme 5) as single diastereoisomers.

\section{Conclusion}

The present work has furnished a facile and efficient stereoselective reduction of isoxazoline-fused cyclic $\beta$-amino esters to multifunctionalized 2-aminocyclopentanecarboxylates through the use of $\mathrm{NaBH}_{4} / \mathrm{NiCl}_{2}$ as reducing agent. As Peramivir related derivatives, highly functionalized cyclic amino esters may be regarded as promising bioactive compounds.<smiles>CCOC1C[C@@H]2ON=C(C)[C@H]2[C@H]1NC(=O)OCc1ccccc1</smiles>

2

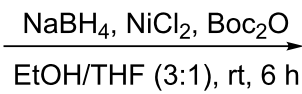

$\mathrm{EtOH} / \mathrm{THF}(3: 1), \mathrm{rt}, 6 \mathrm{~h}$<smiles>CCOC(=O)C1C[C@H](O)[C@H](C(C)NC(=O)OCc2ccccc2)C1NC(=O)OCc1ccccc1</smiles>

$(80 \%)$ 

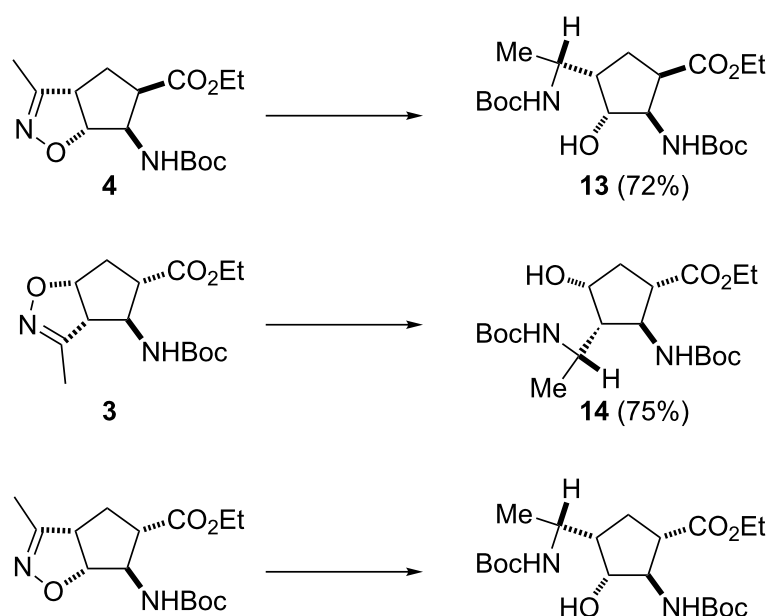

5

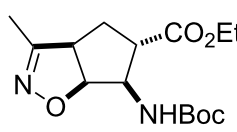

6

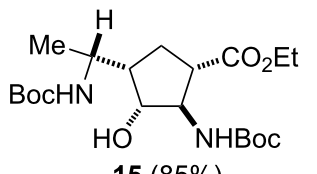

$15(85 \%)$

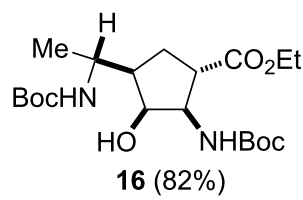

Scheme 5: Synthesis of multifunctionalized $\beta$-amino acid derivatives 13-16. Reaction conditions: $\mathrm{NaBH}_{4}, \mathrm{NiCl}_{2}, \mathrm{Boc}_{2} \mathrm{O}, \mathrm{EtOH} / \mathrm{H}_{2} \mathrm{O}, \mathrm{rt}, 6 \mathrm{~h}$.

\section{Experimental}

The chemicals were purchased from Aldrich. The solvents were used as received from the supplier. Melting points were determined with a Kofler apparatus. NMR spectra were recorded on a Bruker DRX $400 \mathrm{MHz}$ spectrometer in deuterated DMSO or $\mathrm{CDCl}_{3}$. Chemical shifts are expressed in ppm $(\delta)$ from the signal of internal tetramethylsilane. Mass spectra were recorded on a Finnigan MAT 95S spectrometer. Elemental analyses were recorded on a Perkin-Elmer CHNS-2400 Ser II Elemental Analyzer. FTIR spectra were recorded on a Perkin-Elmer Spectrum 100 instrument. Cycloadducts 2-6 were synthesized according to previously published procedures [8].

\section{General procedure for the synthesis of com- pounds 8 and 9}

To a solution of izoxazoline-fused $\beta$-aminocyclopentanecarboxylate $2(0.96 \mathrm{mmol})$ in dry EtOH $(15 \mathrm{~mL}) \mathrm{NaBH}_{4}$ $(2.88 \mathrm{mmol})$ was added and the reaction mixture was stirred under reflux for $16 \mathrm{~h}$. The reaction was quenched by the addition of $\mathrm{H}_{2} \mathrm{O}(10 \mathrm{~mL})$ and then, the mixture was concentrated under reduced pressure. The reaction mixture was diluted with $\mathrm{H}_{2} \mathrm{O}(20 \mathrm{~mL})$, washed with EtOAc $(3 \times 15 \mathrm{~mL})$, dried $\left(\mathrm{Na}_{2} \mathrm{SO}_{4}\right)$ and concentrated under reduced pressure. The crude residue was purified by column chromatography on silica gel ( $n$-hexane/EtOAc) giving 8 and 9.

\section{General procedure for the synthesis of $\mathbf{1 0}$}

To a stirred solution of isoxazoline-fused $\beta$-aminocyclopentanecarboxylate $2(1.6 \mathrm{mmol})$ in dry EtOH $(15 \mathrm{~mL}), \mathrm{HCOONH}_{4}$ $(3.2 \mathrm{mmol})$ and $\mathrm{Pd} / \mathrm{C}(0.10 \mathrm{~g})$ were added and the reaction mixture was stirred under reflux for $24 \mathrm{~h}$. The mixture was filtered through a celite pad and the filtrate was evaporated in vacuo. The crude residue was diluted with EtOAc $(30 \mathrm{~mL})$, washed with $\mathrm{H}_{2} \mathrm{O}(3 \times 15 \mathrm{~mL})$, dried over $\mathrm{Na}_{2} \mathrm{SO}_{4}$ and concentrated under reduced pressure. The residue was purified by column chromatography on silica gel ( $n$-hexane/EtOAc), giving $\mathbf{1 0}$.

\section{General procedure for isoxazoline ring opening}

To a stirred solution of isoxazoline-fused $\beta$-aminocyclopentanecarboxylates 2-6 $(0.96 \mathrm{mmol})$ in $8 \mathrm{~mL}$ of EtOH/THF (v:v = $3: 1), \mathrm{NiCl}_{2}(1.92 \mathrm{mmol})$ and $\mathrm{Boc}_{2} \mathrm{O}(1.92 \mathrm{mmol})$ were added. After stirring for $10 \mathrm{~min}, \mathrm{NaBH}_{4}(1.92 \mathrm{mmol})$ was added in portions. The reaction mixture was further stirred for $6 \mathrm{~h}$ at room temperature and the reaction was quenched by the addition of $\mathrm{H}_{2} \mathrm{O}(5 \mathrm{~mL})$. The reaction mixture was filtered through a celite pad and the filtrate was evaporated in vacuo. The crude residue was diluted with EtOAc $(30 \mathrm{~mL})$, washed with $\mathrm{H}_{2} \mathrm{O}$ $(3 \times 15 \mathrm{~mL})$, dried over $\mathrm{Na}_{2} \mathrm{SO}_{4}$, and concentrated under reduced pressure. The residue was purified by column chromatography on silica gel ( $n$-hexane/EtOAc), giving the corresponding reduced product.

tert-Butyl $(3 \mathrm{a} R *, 4 R *, 5 R *, 6 \mathrm{a} R *)$-[5-(hydroxymethyl)-3methyl-4,5,6,6a-tetrahydro-3a $H$-cyclopenta $[d]$ isoxazol-4yl]carbamate (8): Light-yellow oil; yield 48\% (124 mg); $R_{\mathrm{f}}$ 0.35 ( $n$-hexane/EtOAc); IR (KBr) $v / \mathrm{cm}^{-1}: 3344,3265,2979$, 1678, 1563, 1184; ${ }^{1} \mathrm{H}$ NMR (400 MHz, $\left.\mathrm{CDCl}_{3}\right) \delta 1.45$ (s, 3H, $\left.\mathrm{CH}_{3}\right), 1.56\left(\mathrm{~s}, 9 \mathrm{H}, \mathrm{CH}_{3}\right), 1.65-1.72\left(\mathrm{~m}, 2 \mathrm{H}, \mathrm{CH}_{2}\right), 2.19-2.25$ (m, 1H, H-5), 2.75-2.81 (m, 1H, H-3a), 3.19-3.25 (m, 1H, H-6a), 3.59-3.71 (m, 1H, H-4), 3.63-3.72 (m, 2H, $\left.\mathrm{CH}_{2}\right), 5.42$ (br s, 1H, N-H), OH group not observed - exchanged; ${ }^{13} \mathrm{C}$ NMR $\left(100 \mathrm{MHz}, \mathrm{CDCl}_{3}\right) \delta 16.0,28.6,30.2,32.5,43.0$, 44.4, 59.2, 63.9, 78.0, 155.2, 155.6; MS (ESI) $\mathrm{m} / \mathrm{z}: 293[\mathrm{M}+$ $\mathrm{Na}]^{+}$; Anal. calcd for $\mathrm{C}_{13} \mathrm{H}_{22} \mathrm{~N}_{2} \mathrm{O}_{4}: \mathrm{C}, 57.76 ; \mathrm{H}, 8.20 ; \mathrm{N}, 10.36$; found: $\mathrm{C}, 57.60 ; \mathrm{H}, 8.07 ; \mathrm{N}, 10.23$.

tert-Butyl $\left(3 S^{*}, 3 \mathrm{a} R *, 4 R *, 5 R *, 6 \mathrm{a} R *\right)$-[5-(hydroxymethyl)-3methylhexahydro-2H-cyclopenta $[d]$ isoxazol-4-yl]carbamate (9): Colorless oil; yield 12\% (31 mg); $R_{\mathrm{f}} 0.29$ ( $n$-hexane/ EtOAc); IR (KBr) $v / \mathrm{cm}^{-1}$ : 3460, 3331, 2978, 1683, 1531, 1174; ${ }^{1} \mathrm{H}$ NMR (400 MHz, $\left.\mathrm{CDCl}_{3}\right) \delta$ 0.98-1.05 (m, 3H, $\left.\mathrm{CH}_{3}\right), 1.36$ (s, $\left.9 \mathrm{H}, \mathrm{CH}_{3}\right), 1.55-1.75\left(\mathrm{~m}, 2 \mathrm{H}, \mathrm{CH}_{2}\right), 2.22-2.27$ (m, 1H, H-5), 2.38-2.47 (m, 1H, H-3a), 2.78-2.86 (m, 1H, H-3), 3.17-3.24 (m, 1H, H-6a), 3.59-3.69 (m, 1H, H-4), 3.36-3.68 (m, 2H, $\mathrm{CH}_{2}$ ), 5.32 (br s, 1H, N-H), 6.12 (br s, $\left.1 \mathrm{H}, \mathrm{N}-\mathrm{H}\right), \mathrm{OH}$ group not observed - exchanged; ${ }^{13} \mathrm{C} \mathrm{NMR}\left(100 \mathrm{MHz}, \mathrm{CDCl}_{3}\right) \delta 15.0$, 
27.1, 29.0, 35.8, 42.4, 51.7, 57.2, 62.6, 77.4, 80.4, 155.6; MS (ESI) $m / z: 295[\mathrm{M}+\mathrm{Na}]^{+}$; Anal. calcd for $\mathrm{C}_{13} \mathrm{H}_{24} \mathrm{~N}_{2} \mathrm{O}_{4}$ : C, 57.33; H, 8.88; N, 10.29; found: C, 57.20; H, 8.71; N, 10.42 .

Ethyl $\left(1 R^{*}, 2 S *, 3 S *\right)-3$-acetyl-2-(tert-butoxycarbonylamino)cyclopentanecarboxylate (10): White solid; yield $32 \%$ (153 mg); mp 109-110 ${ }^{\circ} \mathrm{C} ; R_{\mathrm{f}} 0.62$ (n-hexane/EtOAc); IR (KBr) $v / \mathrm{cm}^{-1}: 3354,2978,1716,1684,1531,1171 ;{ }^{1} \mathrm{H}$ NMR $(400$ $\left.\mathrm{MHz}, \mathrm{CDCl}_{3}\right) \delta 1.29\left(\mathrm{t}, J=7.54 \mathrm{~Hz}, 3 \mathrm{H}, \mathrm{CH}_{3}\right), 1.41(\mathrm{~s}, 9 \mathrm{H}$, $\left.\mathrm{CH}_{3}\right), 1.59-1.71\left(\mathrm{~m}, 2 \mathrm{H}, \mathrm{CH}_{2}\right), 1.74-1.95\left(\mathrm{~m}, 2 \mathrm{H}, \mathrm{CH}_{2}\right), 2.05$ (s, 3H, $\left.\mathrm{CH}_{3}\right), 2.83-2.97$ (m, 1H, H-1), 3.01-3.15 (m, 1H, H-3), 4.18-4.29 (m, 2H, $\left.\mathrm{OCH}_{2}\right), 4.31-4.44(\mathrm{~m}, 1 \mathrm{H}, \mathrm{H}-2), 5.76$ (br s, $1 \mathrm{H}, \mathrm{N}-\mathrm{H}) ;{ }^{13} \mathrm{C} \mathrm{NMR}\left(100 \mathrm{MHz}, \mathrm{CDCl}_{3}\right) \delta 13.98,20.05,25.76$, 29.31, 31.21, 43.97, 46.01, 52.70, 82.01, 155.67, 176.01, 206.52; MS (ESI) $\mathrm{m} / z: 322[\mathrm{M}+\mathrm{Na}]^{+}$; Anal. calcd for $\mathrm{C}_{15} \mathrm{H}_{25} \mathrm{NO}_{5}$ : C, 60.18; H, 8.42; N, 4.68; found: $\mathrm{C}, 60.05 ; \mathrm{H}$, $8.35 ; \mathrm{N}, 4.54$.

Ethyl $\left(1 R^{*}, 2 S^{*}, 3 S^{*}, 4 R^{*}\right)-2-\left(\right.$ tert-butoxycarbonyl)-3-(( $\left.S^{*}\right)-1-$ (tert-butoxycarbonyl)ethyl)-4-hydroxycyclopentanecarboxylate (12): White solid; yield 80\% (320 mg); mp 120-121 ${ }^{\circ} \mathrm{C} ; R_{\mathrm{f}}$ 0.22 ( $n$-hexane/EtOAc $1: 1)$; IR (KBr) $v / \mathrm{cm}^{-1}: 3457,3348$, 2982, 1720, 1698, 1531, 1160; ${ }^{1} \mathrm{H}$ NMR (400 MHz, DMSO) $\delta$ $0.96\left(\mathrm{t}, J=7.34 \mathrm{~Hz}, 3 \mathrm{H}, \mathrm{CH}_{3}\right), 1.27-1.33\left(\mathrm{~m}, 3 \mathrm{H}, \mathrm{CH}_{3}\right)$, 1.45-1.50 (m, 18H, CH 3$), 1.94-2.02\left(\mathrm{~m}, 2 \mathrm{H}, \mathrm{CH}_{2}\right), 2.07-2.16$ (m, 1H, H-4), 3.30-3.39 (m, 1H, H-1), 3.80-3.89 (m, 1H, CH), 4.13-4.23 (m, 2H, $\left.\mathrm{OCH}_{2}\right), 4.24-4.30(\mathrm{~m}, 1 \mathrm{H}, \mathrm{H}-2), 4.44-4.56$ (m, 1H, H-3), 5.28-5.35 (m, 1H, NH), 5.61-5.72 (m, 1H, NH), $\mathrm{OH}$ group not observed - exchanged; ${ }^{13} \mathrm{C}$ NMR $(100 \mathrm{MHz}$, $\left.\mathrm{CDCl}_{3}\right) \delta 11.7,14.6,28.8,28.9,29.3,30.1,31.8,37.3,44.6$, 51.1, 54.6, 61.2, 73.7, 80.1, 80.4, 155.0, 156.5, 172.0; MS (ESI) $m / z: 418[\mathrm{M}+2 \mathrm{H}]^{+}$; Anal. calcd for $\mathrm{C}_{20} \mathrm{H}_{36} \mathrm{~N}_{2} \mathrm{O}_{7}: \mathrm{C}, 57.67 ; \mathrm{H}$, 8.71 ; N, 6.73; found: C, 57,44; H, 8.86; N, 6.58.

Ethyl $\left(1 R^{*}, 2 R^{*}, 3 R^{*}, 4 S^{*}\right)-2$-(tert-butoxycarbonyl)-4-(( $\left.R^{*}\right)-1-$ (tert-butoxycarbonyl)ethyl)-3-hydroxycyclopentanecarboxylate (13): White solid; yield 72\% (288 mg); mp 129-130 ${ }^{\circ} \mathrm{C} ; R_{\mathrm{f}}$ 0.59 ( $n$-hexane/EtOAc 1:1); IR (KBr) $v / \mathrm{cm}^{-1}: 3479,3347$, $3353,1725,1685,1662,1531,1163 ;{ }^{1} \mathrm{H}$ NMR $(400 \mathrm{MHz}$, $\left.\mathrm{CDCl}_{3}\right) \delta 1.17-1.29\left(\mathrm{~m}, 6 \mathrm{H}, \mathrm{CH}_{3}\right), 1.40-1.46\left(\mathrm{~m}, 18 \mathrm{H}, \mathrm{CH}_{3}\right)$, 1.79-1.91 (m, 1H, $\left.\mathrm{CH}_{2}\right), 2.05-2.19\left(\mathrm{~m}, 2 \mathrm{H}, \mathrm{CH}_{2}, \mathrm{H}-1\right)$, 3.26-3.34 (m, 1H, H-4), 3.86-4.01 (m, 2H, H-2, CH), $4.08-4.19$ (m, 3H, $\mathrm{OCH}_{2}, \mathrm{H}-3$ ), 4.53 (br s, 1H, N-H), 5.05 (br s, $1 \mathrm{H}, \mathrm{N}-\mathrm{H}), \mathrm{OH}$ group not observed - exchanged; ${ }^{13} \mathrm{C}$ NMR (100 $\left.\mathrm{MHz}, \mathrm{CDCl}_{3}\right) \delta 14.6,21.1,28.4,28.7,28.8,44.0,46.6,60.5$, 61.2, 67.5, 77.6, 80.2, 86.4, 156.1, 156.4, 174.8; MS (ESI) $\mathrm{m} / \mathrm{z}$ : $418[\mathrm{M}+2 \mathrm{H}]^{+}$; Anal. calcd for $\mathrm{C}_{20} \mathrm{H}_{36} \mathrm{~N}_{2} \mathrm{O}_{7}$ : C, 57.67; H, 8.71; $\mathrm{N}, 6.73$; found: C, $57.50 ; \mathrm{H}, 8.98 ; \mathrm{N}, 6.39$.

Ethyl $\left(1 S^{*}, 2 S^{*}, 3 S^{*}, 4 R^{*}\right)$-2-(tert-butoxycarbonyl)-3-((S*)-1(tert-butoxycarbonyl)ethyl)-4-hydroxycyclopentanecarboxy- late (14): White solid; yield 75\% (300 mg); mp 144-145 ${ }^{\circ} \mathrm{C} ; R_{\mathrm{f}}$ 0.3 (n-hexane/EtOAc 1:1); IR (KBr) $v / \mathrm{cm}^{-1}: 3420,3363,2980$, $1692,1537,1185 ;{ }^{1} \mathrm{H} \mathrm{NMR}\left(400 \mathrm{MHz}, \mathrm{CDCl}_{3}\right) \delta 1.26-1.33(\mathrm{~m}$, $\left.6 \mathrm{H}, \mathrm{CH}_{3}\right), 1.43-1.48\left(\mathrm{~m}, 18 \mathrm{H}, \mathrm{CH}_{3}\right), 1.82-1.93\left(\mathrm{~m}, 1 \mathrm{H}, \mathrm{CH}_{2}\right)$, 1.98-2.15 (m, 1H, H-1), 2.24-2.36 (m, 1H, $\left.\mathrm{CH}_{2}\right), 2.76-2.89$ (m, 1H, H-3), 3.58-3.72 (m, 1H, H-4), 3.93-4.05 (m, 1H, H-2), 4.15-4.25 (m, 3H, $\left.\mathrm{OCH}_{2}, \mathrm{CH}\right), 4.87$ (br s, 1H, N-H), 5.09 (br s, $1 \mathrm{H}, \mathrm{N}-\mathrm{H}), \mathrm{OH}$ group not observed - exchanged; ${ }^{13} \mathrm{C}$ NMR (100 $\left.\mathrm{MHz}, \mathrm{CDCl}_{3}\right) \delta 14.5,21.4,28.8,28.9,35.9,45.7,49.1,52.3$, $54.5,58.3,73.4,80.1,152.5,156.8,172.6$; MS (ESI) $m / z: 418$ $[\mathrm{M}+2 \mathrm{H}]^{+}$; Anal. calcd for $\mathrm{C}_{20} \mathrm{H}_{36} \mathrm{~N}_{2} \mathrm{O}_{7}: \mathrm{C}, 57.67 ; \mathrm{H}, 8.71 ; \mathrm{N}$, 6.73; found: C, 57.41; H, 8.37; N, 6.59.

Ethyl $\left(1 S^{*}, 2 R^{*}, 3 R^{*}, 4 S^{*}\right)-2-\left(\right.$ tert-butoxycarbonyl)-4-(( $\left.R^{*}\right)-1-$ (tert-butoxycarbonyl)ethyl)-3-hydroxycyclopentanecarboxylate (15): White solid; yield $85 \%$ (340 mg); mp 141-142 ${ }^{\circ} \mathrm{C} ; R_{\mathrm{f}}$ 0.46 ( $n$-hexane/EtOAc 1:1); IR (KBr) v/ $\mathrm{cm}^{-1}: 3426,3378$, $3333,2979,1688,1718,1703,1522,1176 ;{ }^{1} \mathrm{H}$ NMR (400 $\left.\mathrm{MHz}, \mathrm{CDCl}_{3}\right) \delta 1.21-1.30\left(\mathrm{~m}, 6 \mathrm{H}, \mathrm{CH}_{3}\right), 1.40-1.46(\mathrm{~m}, 18 \mathrm{H}$, $\mathrm{CH}_{3}$ ), 1.84-1.97 (m, 2H, CH, $\left.\mathrm{H}-4\right), 2.03-2.20$ (m, 2H, $\mathrm{CH}_{2}$, $\mathrm{H}-1), 2.54$ (q, $J=9.10 \mathrm{~Hz}, 1 \mathrm{H}, \mathrm{H}-2$ ), 3.73-3.82 (m, 1H, H-3), $3.87-4.04(\mathrm{~m}, 2 \mathrm{H}, \mathrm{N}-\mathrm{H}, \mathrm{CH}), 4.10-4.22\left(\mathrm{~m}, 2 \mathrm{H}, \mathrm{OCH}_{2}\right), 4.83$ (br s, 1H, N-H), OH group not observed - exchanged; ${ }^{13} \mathrm{C}$ NMR $\left(100 \mathrm{MHz}, \mathrm{CDCl}_{3}\right) \delta 14.1,20.0,27.5,28.7,28.8$, 45.6, 46.1, 46.8, 60.9, 62.5, 78.1, 80.1, 80.3, 154.0, 156.4, 174.6; MS (ESI) $m / z: 418[\mathrm{M}+2 \mathrm{H}]^{+}$; Anal. calcd for $\mathrm{C}_{20} \mathrm{H}_{36} \mathrm{~N}_{2} \mathrm{O}_{7}$ : C, 57.67; H, 8.71; N, 6.73; found: $\mathrm{C}, 57.91 ; \mathrm{H}$, $8.46 ; \mathrm{N}, 6.58$.

Ethyl $\left(1 S^{*}, 2 R^{*}, 3 S^{*}, 4 R^{*}\right)-2-\left(\right.$ tert-butoxycarbonyl)-4-(( $\left.S^{*}\right)-1-$ (tert-butoxycarbonyl)ethyl)-3-hydroxycyclopentanecarboxylate (16): White solid; yield $82 \%$ (328 mg); mp 166-167 ${ }^{\circ} \mathrm{C} ; R_{\mathrm{f}}$ 0.32 ( $n$-hexane/EtOAc $1: 1$ ); IR (KBr) $v / \mathrm{cm}^{-1}: 3485,3368$, 3353, 2975, 1733, 1681, 1667, 1533, 1167; ${ }^{1} \mathrm{H}$ NMR (400 $\left.\mathrm{MHz}, \mathrm{CDCl}_{3}\right) \delta 1.17-1.31\left(\mathrm{~m}, 6 \mathrm{H}, \mathrm{CH}_{3}\right), 1.38-1.46(\mathrm{~m}, 18 \mathrm{H}$, $\left.\mathrm{CH}_{3}\right), 1.79-2.15$ (m, 3H, CH $\left.2, \mathrm{H}-1, \mathrm{H}-4\right), 2.72-2.87$ (m, $1 \mathrm{H}$, $\mathrm{CH}_{2}$ ), 3.77-4.03 (m, 1H, CH), 4.06-4.23 (m, 4H, H-2, H-3, $\mathrm{OCH}_{2}$ ), 4.37-4.48 (m, 1H, N-H), 4.88 (br s, 1H, N-H), OH group not observed - exchanged; ${ }^{13} \mathrm{C} \mathrm{NMR}\left(100 \mathrm{MHz}, \mathrm{CDCl}_{3}\right)$ 14.6, 21.6, 28.7, 28.8, 47.2, 49.0, 59.9, 61.2, 61.6, 69.4, 74.7, 80.0, 85.9, 117.5, 156.1, 158.8, 171.3; MS (ESI) $\mathrm{m} / \mathrm{z}: 418[\mathrm{M}+$ $2 \mathrm{H}]^{+}$; Anal. calcd for $\mathrm{C}_{20} \mathrm{H}_{36} \mathrm{~N}_{2} \mathrm{O}_{7}$ : C, 57.67; $\mathrm{H}, 8.71$; N, 6.73; found: $\mathrm{C}, 57.43 ; \mathrm{H}, 8.40 ; \mathrm{N}, 6.95$.

X-ray crystallographic study of 12: Crystallographic data were collected at $123 \mathrm{~K}$ with a Nonius-Kappa CCD area detector diffractometer, using graphite-monochromatized Mo $K_{a}$ radiation $(\lambda=0.71073 \AA)$ as reported earlier [51]. Crystal data for $12, \mathrm{C}_{20} \mathrm{H}_{36} \mathrm{~N}_{2} \mathrm{O}_{7}, M_{r}=416.51$, triclinic, space group $P-1$ (no. 2), $a=9.3765(2), b=13.7078(4), c=$ 18.7792(4) $\AA, \alpha=96.609(2), \beta=95.261(1), \gamma=100.965(1), V=$ 
2337.9(1) $\AA^{3}, T=123 \mathrm{~K}, Z=4, \mu\left(\mathrm{Mo} \mathrm{K}_{\alpha}\right)=0.089 \mathrm{~mm}^{-1}, 9120$ unique reflections $\left(R_{\text {int }}=0.034\right)$ which were used in calculations. The final $R l$ (for the data with $F^{2}>2 \delta\left(F^{2}\right)$ was 0.042 and $w R 2\left(F^{2}\right)$ (all data) was 0.111 .

The SHELXL-97 program [52] was used to solve the structure by direct methods and to perform full-matrix, least-squares refinements on $F^{2}$. The unit cell of $\mathbf{1 2}$ contains two molecules with slightly different conformations. The $\mathrm{CH}$ hydrogen atoms were included at fixed distances from their host atoms with fixed displacement parameters. The $\mathrm{NH}$ and $\mathrm{OH}$ hydrogen atoms were refined isotropically. The deposition number CCDC 845835 contains the supplementary crystallographic data for this paper. These data can be obtained free of charge at http:// www.ccdc.cam.ac.uk/conts/retrieving.html [or from the Cambridge Crystallographic Data Centre, 12 Union Road, Cambridge CB2 1EZ, UK; Fax: (internat.) +44-1223-336-033; Email: deposit@ccdc.cam.ac.uk].

\section{Acknowledgements}

We are grateful to the Hungarian Research Foundation (OTKA No. T81371) for financial support and acknowledge the receipt of a Bolyai János Fellowship for Loránd Kiss. We also thank TAMOP-4.2.1/B-09/1/KONV-2010-0005.

\section{References}

1. Pinto, A.; Conti, P.; De Amici, M.; Tamborini, L.; Grazioso, G.; Colleoni, S.; Mennini, T.; Gobbi, M.; De Micheli, C. Tetrahedron: Asymmetry 2008, 19, 867-875. doi:10.1016/j.tetasy.2008.03.001

2. Conti, P.; Caligiuri, A.; Pinto, A.; Roda, G.; Tamborini, L.; Nielsen, B.; Madsen, U.; Frydenvang, K.; Colombo, A.; De Micheli, C. Eur. J. Med. Chem. 2007, 42, 1059-1068. doi:10.1016/j.ejmech.2007.01.013

3. Roda, G.; Conti, P.; De Amici, M.; He, J.; Polavaropu, P. L.; De Micheli, C. Tetrahedron: Asymmetry 2004, 15, 3079-3090. doi:10.1016/j.tetasy.2004.07.037

4. Conti, P.; De Amici, M.; Di Ventimiglia, S. J.; StensbøI, T. B.; Madsen, U.; Bräuner-Osborne, H.; Russo, E.; De Sarro, G.; Bruno, G.; De Micheli, C. J. Med. Chem. 2003, 46, 3102-3108. doi:10.1021/jm0308085

5. Park, K.-H.; Olmstead, M. M.; Kurth, M. J. J. Org. Chem. 1998, 63, 113-117. doi:10.1021/jo9714831

6. Pinto, A.; Conti, P.; Grazioso, G.; Tamborini, L.; Madsen, U.; Nielsen, B.; De Micheli, C. Eur. J. Med. Chem. 2011, 46, 787-793. doi:10.1016/j.ejmech.2010.12.020

7. Bode, J. W.; Carreire, E. M. Org. Lett. 2001, 3, 1587-1590. doi:10.1021/ol015885d

8. Bode, J. W.; Fraefel, N.; Muri, D.; Carreira, E. M. Angew. Chem., Int. Ed. 2001, 40, 2082-2085. doi:10.1002/1521-3773(20010601)40:11<2082::AID-ANIE2082>3.0.CO $; 2-1$

9. Jiang, D.; Chen, Y. J. Org. Chem. 2008, 73, 9181-9183. doi:10.1021/jo801831c
10. Tang, S.; He, J.; Sun, Y.; He, L.; She, X. J. Org. Chem. 2010, 75, 1961-1966. doi:10.1021/jo1000065

11. Marotta, E.; Micheloni, L. M.; Scardovi, N.; Righi, P. Org. Lett. 2001, 3, 727-729. doi:10.1021/ol0070379

12. Scott, J. P.; Oliver, S. F.; Brands, K. M. J.; Brewer, S. E.; Davies, A. J.; Gibb, A. D.; Hands, D.; Keen, S. P.; Sheen, F. J.; Reamer, R. A.; Wilson, R. D.; Dolling, U.-H. J. Org. Chem. 2006, 71, 3086-3092. doi:10.1021/jo060033i

13. Maimone, T. J.; Shi, J.; Ashida, S.; Baran, P. S. J. Am. Chem. Soc. 2009, 131, 17066-17067. doi:10.1021/ja908194b

14. Minter, A. R.; Fuller, A. A.; Mapp, A. K. J. Am. Chem. Soc. 2003, 125, 6846-6847. doi:10.1021/ja0298747

15. Fuller, A. A.; Chen, B.; Minter, A. R.; Mapp, A. K. J. Am. Chem. Soc. 2005, 127, 5376-5383. doi:10.1021/ja0431713

16. Sewald, N. Angew. Chem., Int. Ed. 2003, 42, 5794-5795. doi:10.1002/anie.200301692

17. Tokizane, M.; Sato, K.; Ohta, T.; Ito, Y. Tetrahedron: Asymmetry 2008, 19, 2519-2528. doi:10.1016/j.tetasy.2008.11.005

18. Palkó, M.; Kiss, L.; Fülöp, F. Curr. Med. Chem. 2005, 12, 3063-3083. doi:10.2174/092986705774933443

19. Kiss, L.; Forró, E.; Fülöp, F. Synthesis of carbocyclic $\beta$-amino acids. In Amino Acids, Peptides and Proteins in Organic Chemistry; Hughes, A. B., Ed.; Wiley-VCH: Weinheim, Germany, 2009; Vol. 1, pp 367-409.

20. Kiss, L.; Fülöp, F. Synlett 2010, 1302-1314. doi:10.1055/s-0029-1219821

21. Fülöp, F. Chem. Rev. 2001, 101, 2181-2204. doi:10.1021/cr000456z 22. Ishikawa, H.; Suzuki, T.; Orita, H.; Uchimaru, T.; Hayashi, Y. Chem.-Eur. J. 2010, 16, 12616-12626. doi:10.1002/chem.201001108

23. Ko, J. S.; Keum, J. E.; Ko, S. Y. J. Org. Chem. 2010, 75, 7006-7009. doi:10.1021/jo101517g

24. Karpf, M.; Trussardi, R. Angew. Chem., Int. Ed. 2009, 48, 5760-5762. doi:10.1002/anie.200901561

25. Satoh, N.; Akiba, T.; Yokoshima, S.; Fukuyama, T. Tetrahedron 2009, 65, 3239-3245. doi:10.1016/j.tet.2008.09.103

26. Ishikawa, H.; Suzuki, T.; Hayashi, Y. Angew. Chem., Int. Ed. 2009, 48, 1304-1307. doi:10.1002/anie.200804883

27. Sullivan, B.; Carrera, I.; Drouin, M.; Hudlicky, T. Angew. Chem., Int. Ed 2009, 48, 4229-4231. doi:10.1002/anie.200901345

28. Trost, B. M.; Zhang, T. Angew. Chem., Int. Ed. 2008, 47, 3759-3761. doi:10.1002/anie.200800282

29. Zhu, S.; Yu, S.; Wang, Y.; Ma, D. Angew. Chem., Int. Ed. 2010, 49, 4656-4660. doi:10.1002/anie.201001644

30. Mohan, S.; McAtamney, S.; Haselhorst, T.; von Itzstein, M.; Pinto, B. M. J. Med. Chem. 2010, 53, 7377-7391. doi:10.1021/jm100822f

31. Kamimura, A.; Nakano, T. J. Org. Chem. 2010, 75, 3133-3136. doi:10.1021/jo1002856

32. Nie, L.-D.; Shi, X.-X.; Ko, K. H.; Lu, W.-D. J. Org. Chem. 2009, 74, 3970-3973. doi:10.1021/jo900218k

33. Osato, H.; Jones, I. L.; Chen, A.; Chai, C. L. L. Org. Lett. 2010, 12, 60-63. doi:10.1021/ol9024716

34. Wena, W.-H.; Wang, S.-Y.; Tsai, K.-C.; Cheng, Y.-S. E.; Yang, A.-S.; Fang, J.-M.; Wong, C.-H. Bioorg. Med. Chem. 2010, 18, 4074-4084. doi:10.1016/j.bmc.2010.04.010

35. Xu, G.; Kiefel, M. J.; Wilson, J. C.; Andrew, P. W.; Oggioni, M. R.; Taylor, G. L. J. Am. Chem. Soc. 2011, 133, 1718-1721. doi:10.1021/ja110733q 
36. Calveras, J.; Nagai, Y.; Sultana, I.; Ueda, Y.; Higashi, T.; Shoji, M.; Sugai, T. Tetrahedron 2010, 66, 4284-4291.

doi:10.1016/j.tet.2010.04.045

37. Honda, T.; Kubo, S.; Masuda, T.; Arai, M.; Kobayashi, Y.; Yamashita, M. Bioorg. Med. Chem. Lett. 2009, 19, 2938-2940. doi:10.1016/j.bmcl.2009.04.067

38. Soulé, J.-F.; Mathieu, A.; Norsikian, S.; Beau, J.-M. Org. Lett. 2010, 12, 5322-5325. doi:10.1021/ol102326b

39. Sorbera, L. A.; Graul, A.; Castaner, J. Drugs Future 2000, 25 , 249-251. doi:10.1358/dof.2000.025.03.565302

40. Babu, Y. S.; Chand, P.; Bantia, S.; Kotian, P.; Dehghani, A.; El-Kattan, Y.; Lin, T.-H.; Hutchison, T. L.; Elliott, A. J.; Parker, C. D.; Ananth, S. L.; Horn, L. L.; Laver, G. W.; Montgomery, J. A. J. Med. Chem. 2000, 43, 3482-3486. doi:10.1021/jm0002679

41. Chand, P.; Kotian, P. L.; Dehghani, A.; El-Kattan, Y.; Lin, T.-H.; Hutchison, T. L.; Babu, Y. S.; Bantia, S.; Elliott, A. J.; Montgomery, J. A. J. Med. Chem. 2001, 44, 4379-4392. doi:10.1021/jm010277p

42. Chand, P.; Babu, Y. S.; Bantia, S.; Rowland, S.; Dehghani, A.; Kotian, P. L.; Hutchison, T. L.; Ali, S.; Brouillette, W.; El-Kattan, Y.; Lin, T.-H. J. Med. Chem. 2004, 47, 1919-1929. doi:10.1021/jm0303406

43. Lü, W. J.; Chen, Y. L.; Ma, W. P.; Zhang, X. Y.; Luan, F.; Liu, M. C.; Chen, X. G.; Hu, Z. D. Eur. J. Med. Chem. 2008, 43, 569-576. doi:10.1016/j.ejmech.2007.04.011

44. Oakley, A. J.; Barrett, S.; Peat, T. S.; Newman, J.; Streltsov, V. A.; Waddington, L.; Saito, T.; Tashiro, M.; McKimm-Breschkin, J. L. J. Med. Chem. 2010, 53, 6421-6431. doi:10.1021/jm100621s

45. Chand, P.; Bantia, S.; Kotian, P. L.; El-Kattan, Y.; Lin, T.-H.; Babu, Y. S. Bioorg. Med. Chem. 2005, 13, 4071-4077. doi:10.1016/j.bmc.2005.03.048

46. Cui, Y.; Jiao, Z.; Gong, J.; Yu, Q.; Zheng, X.; Quan, J.; Luo, M.; Yang, Z. Org. Lett. 2010, 12, 4-7. doi:10.1021/ol902438f

47. Yi, X.; Guo, Z.; Chu, F. M. Bioorg. Med. Chem. 2003, 11, 1465-1474. doi:10.1016/S0968-0896(02)00602-8

48. Kiss, L.; Nonn, M.; Forró, E.; Sillanpää, R.; Fülöp, F. Tetrahedron Lett. 2009, 50, 2605-2608. doi:10.1016/j.tetlet.2009.03.119

49. Nonn, M.; Kiss, L.; Forró, E.; Mucsi, Z.; Fülöp, F. Tetrahedron 2011, 67, 4079-4085. doi:10.1016/j.tet.2011.04.005

50. Jiang, H.; Elsner, P.; Jensen, K. L.; Falcicchio, A.; Marcos, V.; Jørgensen, K. A. Angew. Chem., Int. Ed. 2009, 48, 6844-6848. doi:10.1002/anie.200901446

51. Kanizsai, I.; Szakonyi, Z.; Sillanpää, R.; D'hooghe, M.; De Kimpe, N.; Fülöp, F. Tetrahedron: Asymmetry 2006, 17, 2857-2863. doi:10.1016/j.tetasy.2006.11.006

52. Sheldrick, G. M. Acta Crystallogr., Sect. A 2008, 64, 112-122. doi:10.1107/S0108767307043930

\section{License and Terms}

This is an Open Access article under the terms of the Creative Commons Attribution License

(http://creativecommons.org/licenses/by/2.0), which permits unrestricted use, distribution, and reproduction in any medium, provided the original work is properly cited.

The license is subject to the Beilstein Journal of Organic Chemistry terms and conditions:

(http://www.beilstein-journals.org/bjoc)

The definitive version of this article is the electronic one which can be found at:

doi: $10.3762 /$ bjoc. 8.10 